\title{
An Improved Lattice Hydrodynamic Model by considering the Effect of "Backward-Looking" and Anticipation Behavior
}

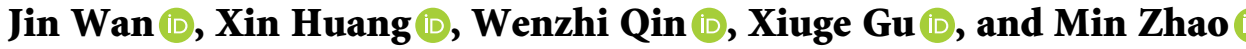 \\ School of Science, Nantong University, Nantong, Jiangsu 226019, China \\ Correspondence should be addressed to Min Zhao; zhao.m@ntu.edu.cn
}

Received 13 July 2021; Revised 7 September 2021; Accepted 13 October 2021; Published 5 November 2021

Academic Editor: Mostafa Al-Emran

Copyright (c 2021 Jin Wan et al. This is an open access article distributed under the Creative Commons Attribution License, which permits unrestricted use, distribution, and reproduction in any medium, provided the original work is properly cited.

\begin{abstract}
In order to prevent the occurrence of traffic accidents, drivers always focus on the running conditions of the preceding and rear vehicles to change their driving behavior. By taking into the "backward-looking" effect and the driver's anticipation effect of flux difference consideration at the same time, a novel two-lane lattice hydrodynamic model is proposed to reveal driving characteristics. The corresponding stability conditions are derived through a linear stability analysis. Then, the nonlinear theory is also applied to derive the $\mathrm{mKdV}$ equation describing traffic congestion near the critical point. Linear and nonlinear analyses of the proposed model show that how the "backward-looking" effect and the driver's anticipation behavior comprehensively affect the traffic flow stability. The results show that the positive constant $\gamma$, the driver's anticipation time $\tau$, and the sensitivity coefficient $p$ play significant roles in the improvement of traffic flow stability and the alleviation of the traffic congestion. Furthermore, the effectiveness of linear stability analysis and nonlinear analysis results is demonstrated by numerical simulations.
\end{abstract}

\section{Introduction}

With the rapid development of wireless technologies and intelligent vehicles in the past years, the vehicle-to-vehicle $(\mathrm{V} 2 \mathrm{~V})$ communication $[1,2]$ can not only alleviate the congested traffic flow but also provide pleasurable driving experiences. To reveal the realistic traffic phenomena, there have emerged various traffic models such as car-following models [3-10], cellular automation models [11, 12], macrotraffic models [13-15], lattice hydrodynamic models [16-21], continuum models [22-24], and gas kinetic models [25].

By incorporating the ideas of car-following as well as continuum models, the first lattice hydrodynamic model [26] with a conservation equation (1) and a flow equation (2) was proposed in 1998.

$$
\begin{gathered}
\partial_{t} \rho_{j}+\rho_{0}\left(\rho_{j} v_{j}-\rho_{j-1} v_{j-1}\right)=0, \\
\partial_{t}\left(\rho_{j} v_{j}\right)=a\left[\rho_{0} V\left(\rho_{j+1}\right)-\rho_{j} v_{j}\right],
\end{gathered}
$$

where $\rho_{0} V\left(\rho_{j+1}\right)$ and $\rho_{j} v_{j}$ denote the optimal flow and the actual flow, respectively. $\rho_{0}$ is the average density, and $a$ refers to the driver's sensitivity. Then, the stability condition and $\mathrm{mKdV}$ equation can be, respectively, obtained with the linear analysis and nonlinear analysis methods. Subsequently, many extended works have been developed by taking different factors into accounts, such as driver's memory [27-31], driver's anticipation effect [32-34], density difference [35], traffic interruption probability [36-38], and "backward-looking" effect [39-41].

In addition to the information from the preceding vehicle, drivers always focus on the running conditions of the rear vehicles from the rear-view mirror to change their driving behavior. By considering the "backward-looking" effect, Ge and Cheng [39] presented the BL-LV model as follows:

$$
\begin{aligned}
& \rho_{j}(t+\tau) v_{j}(t+\tau)=\rho_{0} V_{F}\left(\rho_{j+1}(t)\right) \\
& \quad+H\left(\bar{\rho}-\rho_{j-1}(t)\right) \cdot H\left(\rho_{j-1}(t)-\rho_{c}\right) \rho_{0} V_{B}\left(\rho_{j-1}(t)\right),
\end{aligned}
$$


where $H(\cdot)$ and $\rho_{c}$ represent the Heaviside function and the safety density, respectively. $\bar{\rho}$ denotes a parameter close to 1 . $V_{F}(\cdot)$ is the optimal velocity function for "forward-looking." While $V_{B}(\cdot)$ is the optimal velocity function for "backwardlooking," which works if and only if $\rho_{c} \leq \rho_{j-1}(t) \leq \bar{\rho}$ holds. Functions $V_{F}(\cdot)$ and $V_{B}(\cdot)$ have exactly the opposite effects and are defined as

$$
\begin{aligned}
& V_{F}\left(\rho_{j+1}\right)=\tanh \left(\frac{2}{\rho_{0}}-\frac{\rho_{j+1}}{\rho_{0}^{2}}-\frac{1}{\rho_{c}}\right)+\tanh \left(\frac{1}{\rho_{c}}\right), \\
& V_{B}\left(\rho_{j-1}\right)=\gamma\left[-\tanh \left(\frac{2}{\rho_{0}}-\frac{\rho_{j-1}}{\rho_{0}^{2}}-\frac{1}{\rho_{c}}\right)+\tanh \left(\frac{1}{\rho_{c}}\right)\right],
\end{aligned}
$$

where $\gamma$ is a positive constant that represents the relative role of the "backward-looking."

For the sake of safe and comfortable driving, drivers always predict the velocity of the preceding vehicles so that they can adjust their driving velocity at the next moment. This driving behavior is known as the anticipation effect [42] or predictive effect [43, 44]. Wang et al. [43] added the driver's anticipation effect into the optimal velocity $(\mathrm{OV})$ function in a single-lane lattice model. Later, Kaur and Sharma [44] extended the singlelane model [43] to the two-lane case along with the optimal current difference (OCD) effect. By considering the anticipation effects in the OCD term as well as the OV function, Sharma [45] constructed a new lattice hydrodynamic model with an anticipation coefficient $\alpha$, where the positive $\alpha$ represents the anticipation effect while the negative $\alpha$ represents the delay effect. Then, Li et al. [32] adopted this anticipation effect to the multiple optimal current differences' anticipation version. Meanwhile, numerical results indicated that only the information of three preceding lattices [46] was enough for the improvement of traffic stability. In view of the anticipation effect of flux difference, Chang and Cheng [47] proposed a new lattice model as follows:

$$
\begin{aligned}
\partial_{t}\left(\rho_{j} v_{j}\right)= & a \rho_{0} V\left(\rho_{j+1}\right)-a\left(1+k_{1}\right) \rho_{j} v_{j} \\
& +k_{2}\left[\rho_{j+1}(t+\tau) v_{j+1}(t+\tau)-\rho_{j} v_{j}\right]
\end{aligned}
$$

where $\tau$ represents the driver's anticipation time and $k_{1}$ and $k_{2}$ denote the deviation degree of speed $v_{j}$ and the feedback gain, respectively. Some previous studies have considered the "backward-looking" effect and the anticipation effect of flux difference separately. In a real traffic system, the velocity of the current vehicles is not only affected by the preceding vehicles but also affected by the rear vehicles. Generally, the information of the rear vehicles could be received by the rear-view mirror. According to the information, drivers could change their driving behavior to avoid the traffic accidents. Meanwhile, the velocity of the preceding vehicles could be predicted by drivers on the basis of the vehicle distance information, so that they can adjust their driving velocity in a period of anticipation time. However, the above two factors have never been considered simultaneously in the existing lattice models. This motivates us to develop a new lattice model by incorporating the "backward-looking" effect with the anticipation effect of flux difference.

The outline of this paper is organized as follows. In Section 2, we introduce a new lattice hydrodynamic model considering the flux anticipation difference effect and the "backward-looking" effect and use the linear stability theory to derive the stability condition of the traffic flow. In Section 3 , the propagation behavior of traffic density waves is presented by means of the nonlinear analysis method. In Section 4, numerical simulations are carried out to validate the results of linear and nonlinear stability analyses. At last, the conclusions are drawn in Section 5.

\section{The Extended Lattice Hydrodynamic Model and Linear Stability Analysis}

In light of the aforementioned facts, a novel lattice model is presented by taking the flux anticipation difference effect and the "backward-looking" effect (FADBE) into account as follows:

$$
\begin{aligned}
\partial_{t}\left(\rho_{j} v_{j}\right)= & a\left[\rho_{0} V_{F}\left(\rho_{j+1}(t)\right)+H\left(\bar{\rho}-\rho_{j-1}(t)\right) \cdot H\left(\rho_{j-1}(t)-\rho_{c}\right) \rho_{0} V_{B}\left(\rho_{j-1}(t)\right)\right] \\
& -a \rho_{j} v_{j}+a p\left(\rho_{j+1}(t+\tau) v_{j+1}(t+\tau)-\rho_{j} v_{j}\right),
\end{aligned}
$$

where $p$ is the sensitivity coefficient of the anticipation flux difference. The greater $p$ is, the stronger the driver's anticipation effect is. $\tau$ means the anticipation time, and $\rho_{j+1}(t+\tau) v_{j+1}(t+\tau)-\rho_{j} v_{j}$ represents the anticipation flux difference between the preceding lattice $j+1$ and the current lattice $j$. The optimal velocity functions and the function $H(\cdot)$ are the same as those in Ge and Cheng's model [39].

After eliminating the velocity term $v_{j}$ in equations (1) and (6), the following density equation can be derived: 


$$
\begin{aligned}
& \partial_{t}^{2} \rho_{j}(t)+a \rho_{0}^{2}\left[V\left(\rho_{j+1}(t)\right)-V_{F}\left(\rho_{j}(t)\right)+V_{B}\left(\rho_{j-1}(t)\right)-V_{B}\left(\rho_{j-2}(t)\right)\right] \\
& \quad+a \partial_{t} \rho_{j}+a p\left(\partial_{t} \rho_{j}-\partial_{t} \rho_{j+1}(t+\tau)\right)=0 .
\end{aligned}
$$

When $p=0$, the model reduces to Ge and Cheng's model [39]. In addition, the lattice model coincides with Nagatani's model [26] when $p=0$ and $\tau=0$.

The influence of the "backward-looking" effect and the anticipation effect has been studied by linear stability analysis. The uniform traffic stream with the fixed density $\rho_{0}$ and the optimal velocity $V\left(\rho_{0}\right)$ is defined as the stable state. One can obtain the solution of the steady-state vehicular system as follows:

$$
\begin{aligned}
\rho_{j}(t) & =\rho_{0}, \\
v(j) & =V_{F}(\rho)+V_{B}(\rho) .
\end{aligned}
$$

Then, $y_{j}$ is supposed as a small perturbation added into the steady-state solution at lattice $j$, and the perturbed solution is

$$
\rho_{j}(t)=\rho_{0}+y_{i}(t) .
$$

Equation (9) is substituted into equation (7), and the derived equation is linearized. The equation is obtained as

$$
\begin{aligned}
& \partial_{t}^{2} y_{j}(t)+a \rho_{0}^{2} V_{F}^{\prime}\left(\rho_{0}\right)\left(y_{j+1}(t)-y_{j}(t)\right) \\
& \quad+a \rho_{0}^{2} V_{B}^{\prime}\left(\rho_{0}\right)\left(y_{j-1}(t)-y_{j-2}(t)\right) \\
& \quad-a p \partial_{t} y_{j+1}(t+\tau)+(a p+a) \partial_{t} y_{j}=0 .
\end{aligned}
$$

Let the small perturbation $y_{j}(t)=\exp (i k j+z t)$, and inserting it into equation (10), we gain

$$
\begin{aligned}
z^{2} & +a \rho_{0}^{2}\left[V_{F}^{\prime}\left(\rho_{0}\right)\left(e^{i k}-1\right)+V_{B}^{\prime}\left(\rho_{0}\right)\left(e^{i k}-e^{-2 i k}\right)\right] \\
& -a p z e^{z \tau+i k}+(a p+a) z=0 .
\end{aligned}
$$

Assuming $z=z_{1}(i k)+z_{2}(i k)^{2}+\cdots$ and putting it into equation (11), we reserve the first-order and second-order terms of $i k$ by neglecting higher-order terms. Then, one can get

$$
\begin{aligned}
& z_{1}=-\rho_{0}^{2}\left(V_{F}^{\prime}\left(\rho_{0}\right)+V_{B}^{\prime}\left(\rho_{0}\right)\right) \\
& z_{2}=\frac{2 a p z_{1}-2 z_{1}^{2}+2 a p z_{1}^{2} \tau-a V_{F}^{\prime}\left(\rho_{0}\right) \rho_{0}^{2}+3 a V_{B}^{\prime}\left(\rho_{0}\right) \rho_{0}^{2}}{2 a} .
\end{aligned}
$$

The uniform steady-state flow remains stable as $z_{2}$ is positive. On the contrary, the uniform flow tends to be unstable if the value of $z_{2}$ is negative. When $z_{2}$ is equal to zero, the formula of the neutral stability condition is acquired as

$$
a=\frac{2\left(V_{F}^{\prime}\left(\rho_{0}\right)+V_{B}^{\prime}\left(\rho_{0}\right)\right)^{2} \rho_{0}^{2}}{M},
$$

where $M=-V_{F}^{\prime}\left(\rho_{0}\right)+3 V_{B}^{\prime}\left(\rho_{0}\right)-2\left(V_{F}^{\prime}\left(\rho_{0}\right)+V_{B}^{\prime}\left(\rho_{0}\right)\right) p+2$ $\left(V_{F}^{\prime}\left(\rho_{0}\right)+V_{B}^{\prime}\left(\rho_{0}\right)\right)^{2} p \tau \rho_{0}^{2}$. The stability condition for the uniform traffic flow is obtained as

$$
a>\frac{2\left(V_{F}^{\prime}\left(\rho_{0}\right)+V_{B}^{\prime}\left(\rho_{0}\right)\right)^{2} \rho_{0}^{2}}{M} .
$$

It is obvious that the stable condition (14) is reduced to that of Ge and Cheng's model [39] when $p=0$. Equation (14) shows that the parameters $p, \tau$, and $\gamma$ play important roles in the performance of the traffic system.

The phase diagram of $(\rho, a)$ is shown in Figure 1, where $\rho$ is density and $a$ denotes sensitivity. Note that $\gamma$ denotes a positive constant considering the "backward-looking" effect $[40,41]$. Three solid curves in each pattern of Figure 1 represent the neutral stability curves under $\gamma=0.1$. The phase diagram is divided into two regions: the stable and unstable regions are above and below the solid neutral stability curve, respectively. In the stable region, the traffic flow will remain stable with a perturbation. In the unstable region, the congested traffic will happen even with a small perturbation. Figure 1 depicts the neutral stability curves of the FADBE model for different values of driver's anticipation time $\tau$ and the sensitivity coefficient $p$ of the anticipation flux difference when $\gamma=0.1$. As shown in Figure 1, the corresponding stable region expands gradually with the increasing values of $\tau$ and $p$, which means that the driver's anticipation effect can contribute to the stabilization of traffic flow obviously.

Figure 2 demonstrates the neutral stability curves of the FADBE model for different values of $\tau$ and $\gamma$ when $p=0.1$. As shown in Figure 2, the corresponding neutral stability curves and critical points decrease gradually with the increasing values of $\tau$ and $\gamma$. When the value of $\gamma$ is fixed in each pattern of Figure 2, the stability of the traffic flow can be improved efficiently with the increasing value of anticipation time $\tau$.

Figure 3 exhibits that the phase diagram of $(\rho, a)$ of the FADBE model for different values of the positive constant $\gamma(\gamma=0.05,0.1,0.15,0.2)$ and the sensitivity coefficient $p(p=0.1,0.2,0.3)$ when $\tau=1.5$. With the increasing value of the positive constant $\gamma$ in each pattern of Figure 3, the amplitudes of neutral stability curves all fall down gradually when the value of the parameter $p$ is fixed. For different values of $\gamma$, it can be seen from each pattern of Figure 3 that the corresponding peak value of solid curves lowers down with the increasing value of $p$. These phenomena demonstrate that when the value of $\gamma$ or $p$ increases, the area of the stable region becomes larger. Thus, it can be concluded that the traffic flow stability can be improved by incorporating the positive constant $\gamma$ and the sensitivity coefficient $p$.

\section{Nonlinear Stability Analysis}

To investigate the influence of the "backward-looking" effect and the anticipation effect of flux difference, we carried out the reduction perturbation method to obtain the $\mathrm{mKdV}$ equation. The slow variables $X$ and $T$ are defined as follows: 


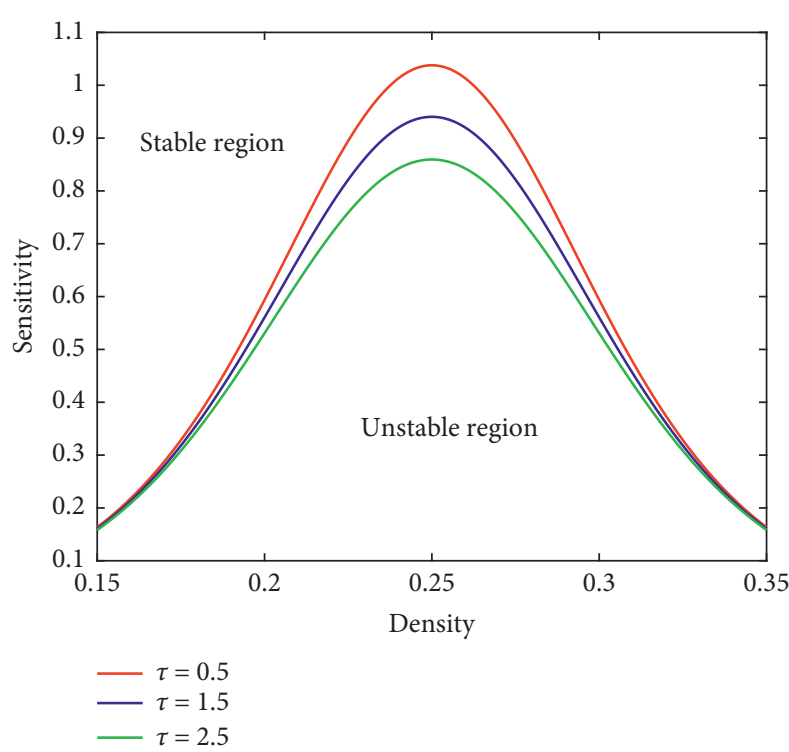

(a)
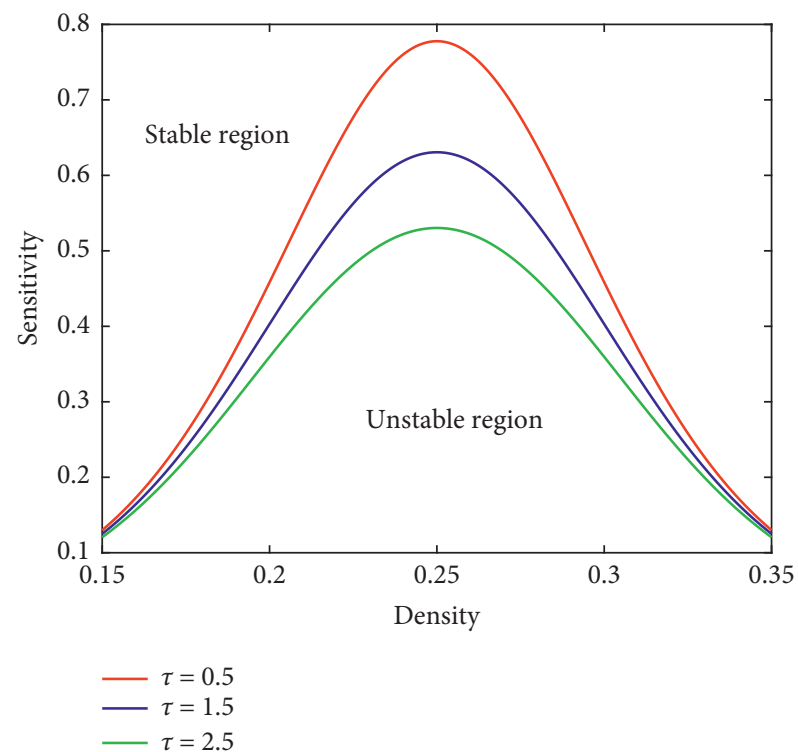

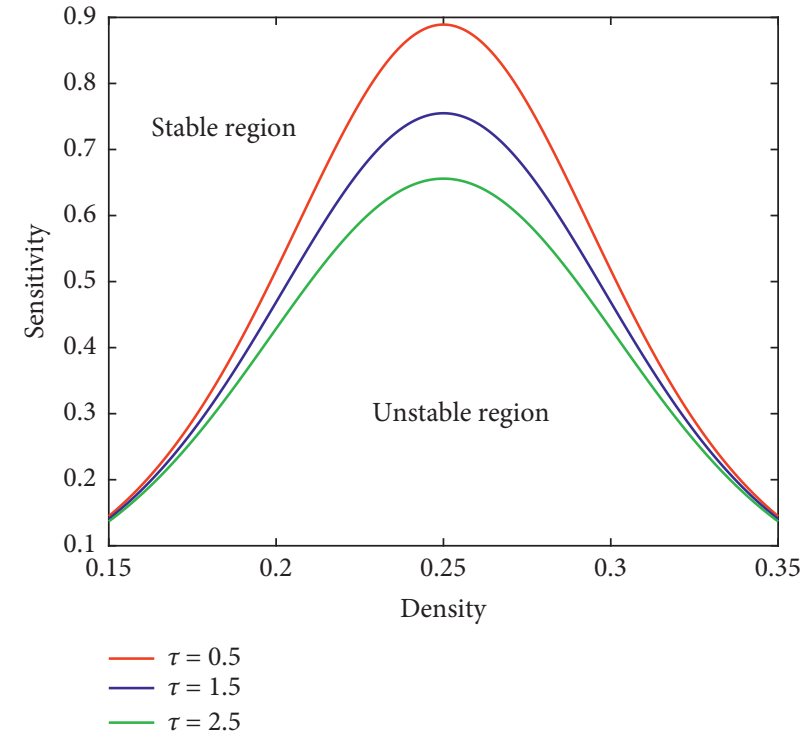

(b)

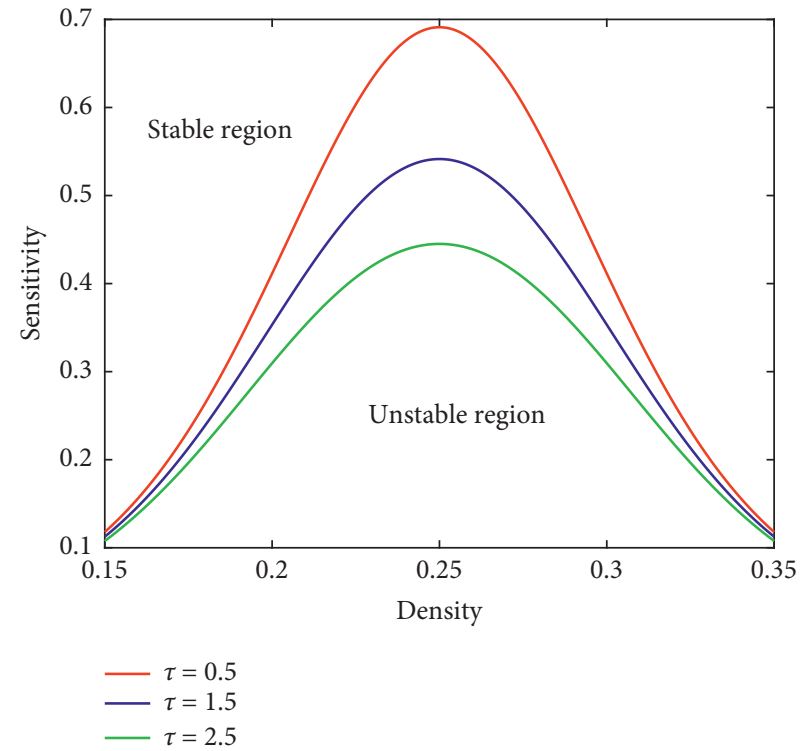

(d)

Figure 1: The neutral stability curves for $\gamma=0.1$ for different values of $p$ and $\tau$ : (a) $p=0.1$; (b) $p=0.2$; (c) $p=0.3$; (d) $p=0.4$.

$$
\begin{aligned}
X & =\varepsilon(j+b t), \\
T & =\varepsilon^{3} t, \\
\rho_{j} & =\rho_{c}+\varepsilon R(X, T),
\end{aligned}
$$

where $\varepsilon(0<\varepsilon \ll 1)$ is a small positive scaling parameter.

Substituting equation (15) into equation (7), each term of equation (7) is expanded up to the fifth-order of $\varepsilon$ with the Taylor expansion technique as follows: 

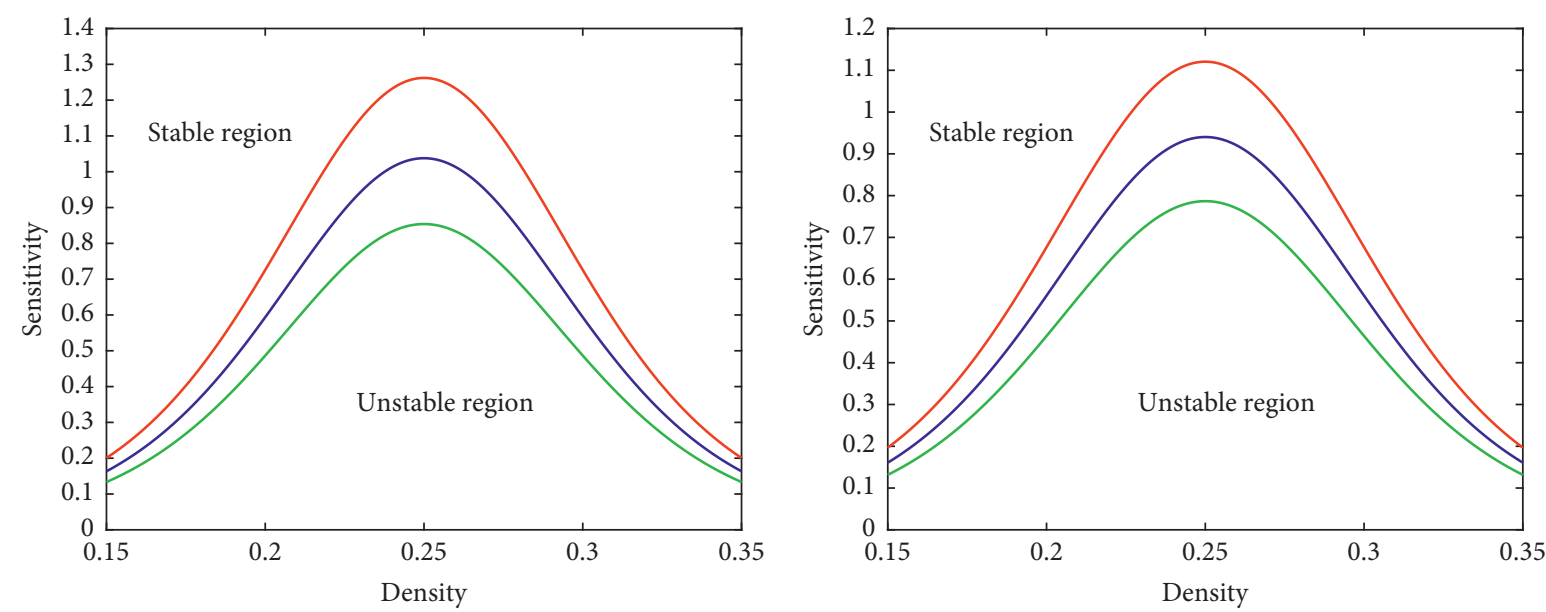

$$
\begin{aligned}
-\gamma & =0.05 \\
-\gamma & =0.1 \\
-\gamma & =0.15
\end{aligned}
$$

$$
\begin{aligned}
-\gamma & =0.05 \\
-\gamma & =0.1 \\
-\gamma & =0.15
\end{aligned}
$$

(a)

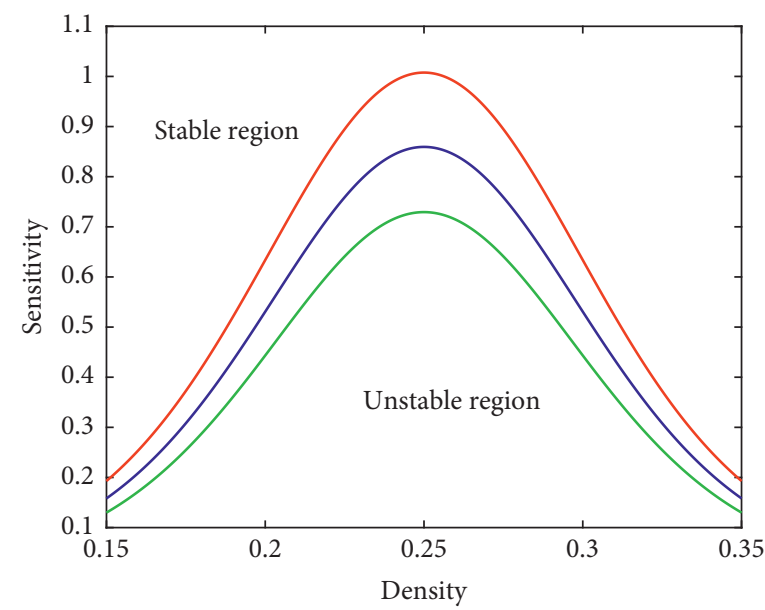

(b)

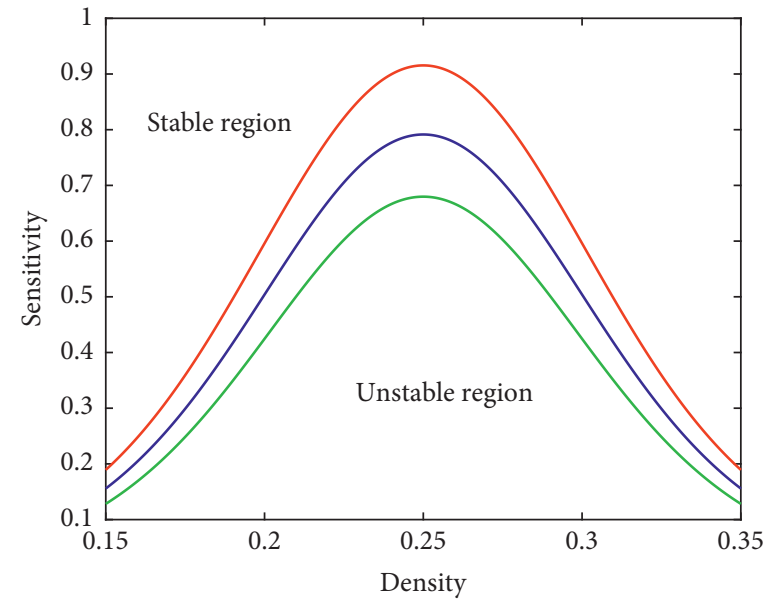

$$
\begin{aligned}
-\gamma=0.05 & -\gamma=0.05 \\
-\gamma=0.1 & -\gamma=0.1 \\
-\gamma=0.15 & -\gamma=0.15
\end{aligned}
$$

(c)

(d)

Figure 2: The neutral stability curves for $p=0.1$ for different values of $\tau$ and $\gamma$ : (a) $\tau=0.5$; (b) $\tau=1.5$; (c) $\tau=2.5$; (d) $\tau=3.5$.

$$
\begin{aligned}
& \varepsilon^{2}\left[b+\rho_{c}^{2}\left(V_{F}^{\prime}+V_{B}^{\prime}\right)\right] \partial_{X} R \\
& +\varepsilon^{3}\left(\frac{b^{2}}{a}-b^{2} p \tau-\frac{3}{2} \rho_{c}^{2} V_{B}^{\prime}+\frac{1}{2} \rho_{c}^{2} V_{F}^{\prime}\right) \partial_{X}^{2} R \\
& +\varepsilon^{4}\left[\partial_{T} R-\left(\frac{1}{2} b^{3} p \tau^{2}-\frac{7}{6} \rho_{c}^{2} V_{B}^{\prime}-\frac{1}{6} \rho_{c}^{2} V_{F}^{\prime}\right) \partial_{X}^{3} R+\left(\frac{1}{6} \rho_{c}^{2} V_{B}^{\prime \prime}+\frac{1}{6} \rho_{c}^{2} V_{f}^{x 2032^{\prime \prime} ;}\right) \partial_{X} R^{3}\right] \\
& +\varepsilon^{5}\left[\left(\frac{2 b}{a}-2 b p \tau\right) \partial_{X} \partial_{T} R+\left(-\frac{1}{6} b^{4} p \tau^{3}-\frac{5}{8} \rho_{c}^{2} V_{B}^{\prime}+\frac{1}{24} \rho_{c}^{2} V_{F}^{\prime}\right) \partial_{X}^{4} R+\left(\frac{1}{12} \rho_{c}^{2} V_{f}^{\prime \prime}-\frac{1}{4} \rho_{c}^{2} V_{B}^{\prime \prime}\right) \partial_{X}^{2} R^{3}\right]=0 \text {, }
\end{aligned}
$$



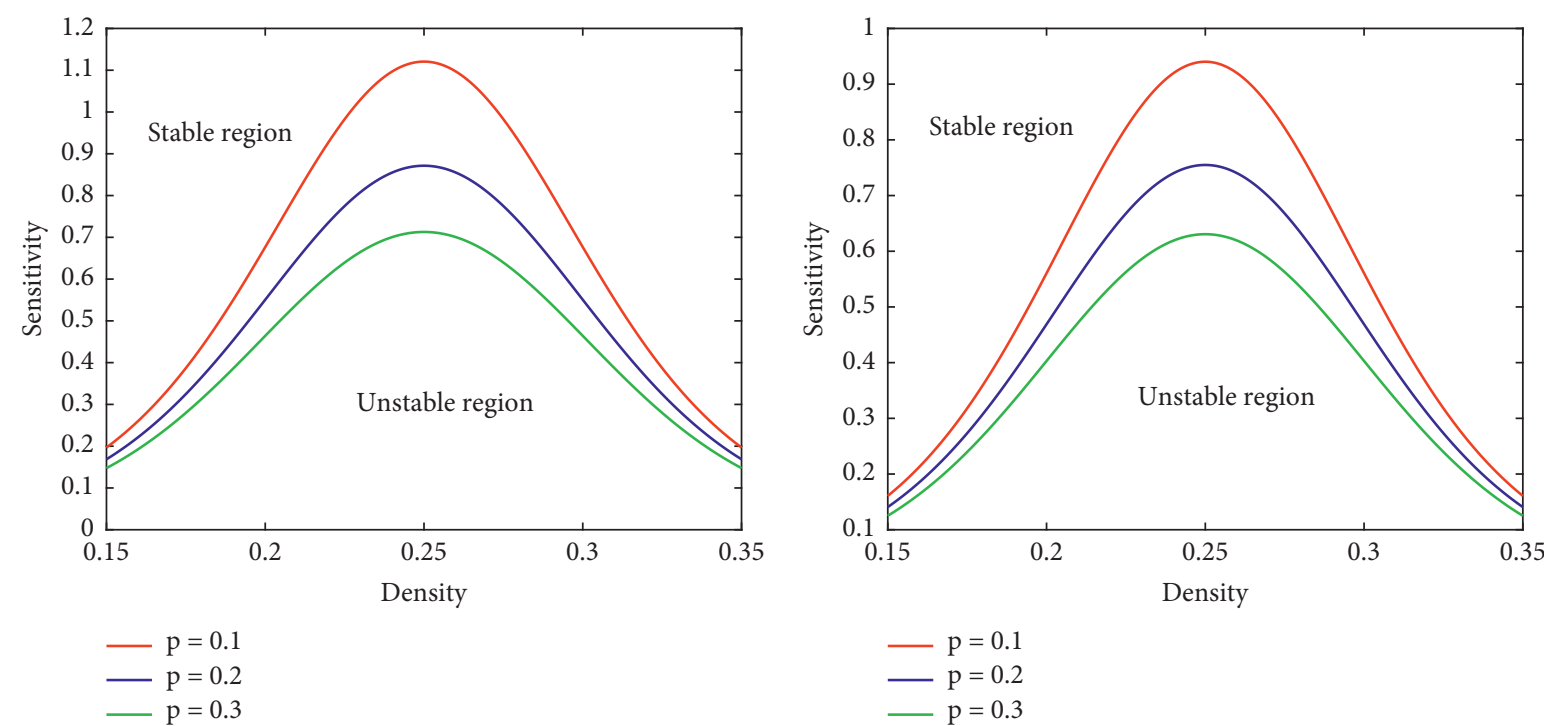

(a)
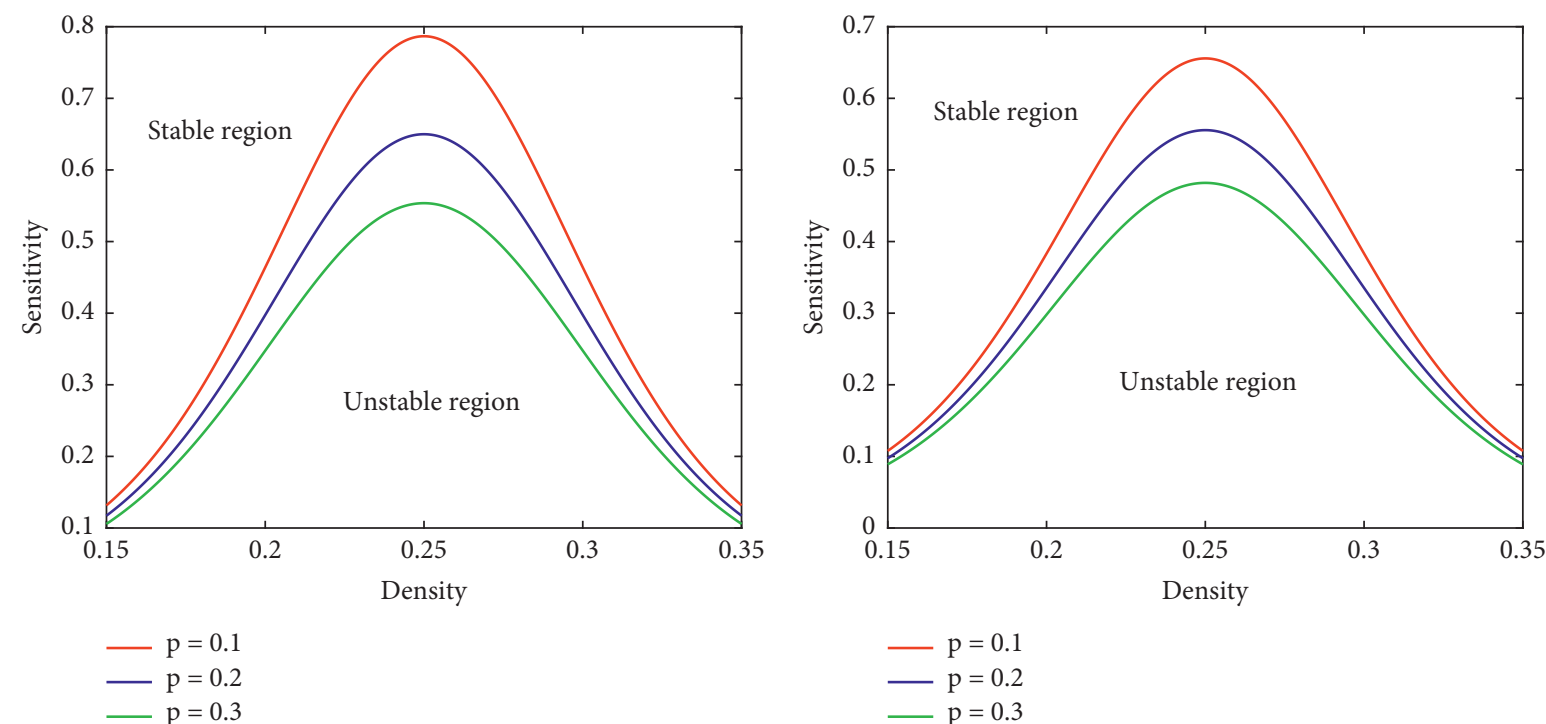

(c)

(d)

Figure 3: The neutral stability curves for $\tau=1.5$ for different values of $\gamma$ and $p$ : (a) $\gamma=0.05$; (b) $\gamma=0.1$; (c) $\gamma=0.15$; (d) $\gamma=0.2$.

where $\quad\left\{\begin{array}{l}V_{F}^{\prime}=\left.(\mathrm{d} V(\rho) / \mathrm{d} \rho)\right|_{\rho=\rho_{c}} \\ V_{F}^{\prime \prime}=\left.\left(\mathrm{d}^{3} V(\rho) / \mathrm{d} \rho^{3}\right)\right|_{\rho=\rho_{c}}\end{array}\right.$

$\left\{\begin{array}{l}V_{B}^{\prime}=\left.(\mathrm{d} V(\rho) / \mathrm{d} \rho)\right|_{\rho=\rho_{c}} \\ V_{B}^{\prime \prime}=\left.\left(\mathrm{d}^{3} V(\rho) / \mathrm{d} \rho^{3}\right)\right|_{\rho=\rho_{c}}\end{array}\right.$. Near the critical point $\left(\rho_{c}, a_{c}\right)$, the value of $a_{c}$ is defined as $a_{c}=a\left(1+\varepsilon^{2}\right)$. The squared and cubic terms of $\varepsilon$ are eliminated in equation (16) with the consideration of $b=-\rho_{c}^{2}\left(V_{F}^{\prime}+V_{B}^{\prime}\right)$. One can get the simplified equation as follows:

$$
\begin{aligned}
\varepsilon^{4} & {\left[\partial_{T} R-g_{1} \partial_{X}^{3} R+g_{2} \partial_{X} R^{3}\right] } \\
& +\varepsilon^{5}\left[g_{3} \partial_{X}^{2} R+g_{4} \partial_{X}^{4} R+g_{5} \partial_{X}^{2} R^{3}\right]=0
\end{aligned}
$$

where 


$$
\begin{aligned}
g_{1}= & -\frac{1}{6} \rho_{c}^{2}\left(7 V_{B}^{\prime}+V_{F}^{\prime}+3 p \rho_{c}^{4} \tau^{2}\left(V_{B}^{\prime}+V_{F}^{\prime}\right)^{3}\right), \\
g_{2}= & \rho_{c}^{2}\left(V_{F}^{\prime \prime}+V_{B}^{\prime \prime}\right), \\
g_{3}= & \frac{1}{2} \rho_{c}^{2}\left(3 V_{B}^{\prime}-V_{F}^{\prime}-2 p\left(V_{B}^{\prime}+V_{F}^{\prime}\right)+2 p \rho_{c}^{2} \tau\left(V_{B}^{\prime}+V_{F}^{\prime}\right)^{2}\right), \\
g_{4}= & -\frac{1}{24} \rho_{c}^{2}\left(15 V_{B}^{\prime}-V_{F}^{\prime}+4 p \rho_{c}^{6} \tau^{3}\left(V_{B}^{\prime}+V_{F}^{\prime}\right)^{4}\right)-\frac{1}{3 a} \rho_{c}^{4}(a p \tau-1)\left(V_{B}^{\prime}+V_{F}^{\prime}\right)\left(3 p \rho_{c}^{4} \tau^{2}\left(V_{B}^{\prime}\right)^{3}+V_{F}^{\prime}\right) \\
& -\frac{1}{3 a} \rho_{c}^{4}(a p \tau-1)\left(V_{B}^{\prime}+V_{F}^{\prime}\right)\left(3 p \rho_{c}^{4} \tau^{2}\left(V_{F}^{\prime}\right)^{3}+V_{B}^{\prime}\left(7+9 p \rho_{c}^{4} \tau^{2}\left(V_{F}^{\prime}\right)^{2}\right)\right), \\
g_{5}= & -\frac{1}{12} \rho_{c}^{2}\left(3 V_{B}^{\prime \prime}-V_{F}^{\prime \prime}\right)-\frac{1}{3 a} \rho_{c}^{4}(a p \tau-1)\left(V_{B}^{\prime}+V_{F}^{\prime}\right)\left(V_{B}^{\prime \prime \prime}+V_{F}^{\prime \prime \prime}\right) .
\end{aligned}
$$

To derive the standard $\mathrm{mKdV}$ equation, the following transformations are applied:

$$
\begin{aligned}
& T=\frac{1}{g_{1}} T^{\prime}, \\
& R=\sqrt{\frac{g_{1}}{g_{2}}} R^{\prime} .
\end{aligned}
$$

Thus, equation (17) is converted into the standard $\mathrm{mKdV}$ equation as follows:

$$
\partial_{T^{\prime}} R^{\prime}=\partial_{X}^{3} R^{\prime}-\partial_{X} R^{\prime 3}+\varepsilon\left[\frac{g_{3}}{g_{1}} \partial_{X}^{2} R^{\prime}+\frac{g_{4}}{g_{1}} \partial_{X}^{4} R^{\prime}+\frac{g_{5}}{g_{2}} \partial_{X}^{2} R^{\prime 3}\right] .
$$

When the term $O(\varepsilon)$ is ignored, the kink-antikink soliton solution of the $\mathrm{mKdV}$ equation can be rewritten as

$$
R_{0}^{\prime}\left(X, T^{\prime}\right)=\sqrt{c} \tanh \left(\sqrt{\frac{c}{2}}\left(X-c T^{\prime}\right)\right)
$$

where $c$ represents the propagation velocity. The specific value of $c$ can be provided only when the following solvability condition is satisfied:

$$
\left(R_{0}^{\prime}, M\left[R_{0}^{\prime}\right]\right) \equiv \int_{-\infty}^{\infty} \mathrm{dX} R_{0}^{\prime} M\left[R_{0}^{\prime}\right]=0
$$

where $\quad M\left[R_{0}^{\prime}\right]=\left(1 / g_{1}\right)\left[g_{3} \partial_{X}^{2} R^{\prime}+g_{4} \partial_{X}^{4} R^{\prime}+\left(g_{1} g_{5} / g_{2}\right)\right.$ $\partial_{X}^{2} R^{\prime 3}$. By solving equation (22), the general solution of propagation velocity $c$ can be calculated as

$$
c=\frac{5 g_{2} g_{3}}{2 g_{2} g_{4}-3 g_{1} g_{5}} \text {. }
$$

Hence, the corresponding kink-antikink solution is derived as

$$
\rho_{j}=\rho_{c}+\varepsilon \sqrt{\frac{g_{1} c}{g_{2}}} \tanh \left(\sqrt{\frac{c}{2}}\left(X-c g_{1} T\right)\right),
$$

where $\varepsilon^{2}=\left(\left(a_{c} / a\right)-1\right)$, and the amplitude of the density wave $A$ is defined as $\sqrt{\left(g_{1} \varepsilon^{2} C / g_{2}\right)}$. According to the kinkantikink solution equation (24), one can get the coexisting phases which include the freely moving phase with low density $\left(\rho_{j}=\rho_{c}-A\right)$ and the congested phase with high density $\left(\rho_{j}=\rho_{c}+A\right)$.

Based on the linear and nonlinear theory analyses, numerical simulations are carried out with the comprehensive consideration of the anticipation effect of flux difference and the "backward-looking" effect.

\section{Numerical Simulations}

As a positive constant $\gamma$ considering the "backward-looking" effect has been studied fully in some previous studies $[36,40,41]$, this paper will focus on the effects of parameters $p$ and $\tau$ in this part. Periodic boundary conditions are adopted by a step function as follows:

$$
\begin{aligned}
& \rho_{j}(0)=\rho_{0}=0.25, \quad j \in[1, N], \\
& \rho_{j}(1)= \begin{cases}\rho_{0} ; & j \neq \frac{N}{2}, \frac{N}{2}-1, \\
\rho_{0}+\Delta \rho ; & j=\frac{N}{2}-1, \\
\rho_{0}-\Delta \rho ; & j=\frac{N}{2},\end{cases}
\end{aligned}
$$

where $\Delta \rho=0.01$ is the initial perturbation and other parameters are set as $N=200, a=1.2$, and $\rho_{0}=\rho_{c}=0.25$.

4.1. The Effect of Anticipation Time $\tau$. In order to analyze the anticipation time's influence on the stability of the traffic system, the temporal and spatial evolution of density waves between time $t=10000-10200 \mathrm{~s}$ is depicted in Figure 4 for different anticipation time $\tau$ with fixed parameters $p=0.1$ and $\gamma=0.05$. Since the given parameters in Figures 4(a)-4(c) do not satisfy the linear stable condition equation (14), kinkantikink density waves appear and the initial stable traffic flow evolves into nonuniform traffic flow with the added perturbation. To reveal the amplitude of the density wave more clearly, Figure 5 describes the density profile of each 


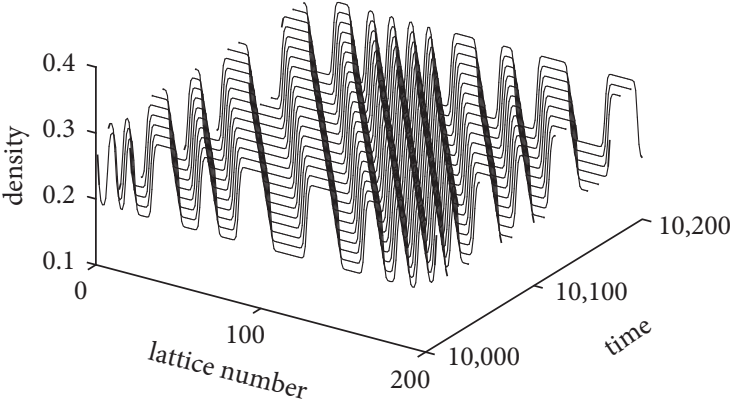

(a)

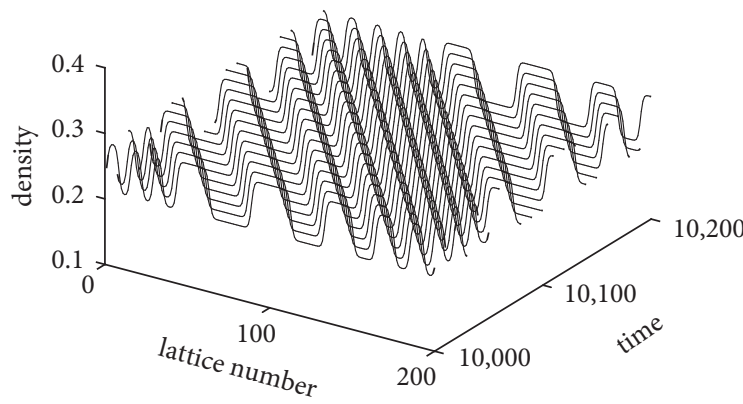

(c)

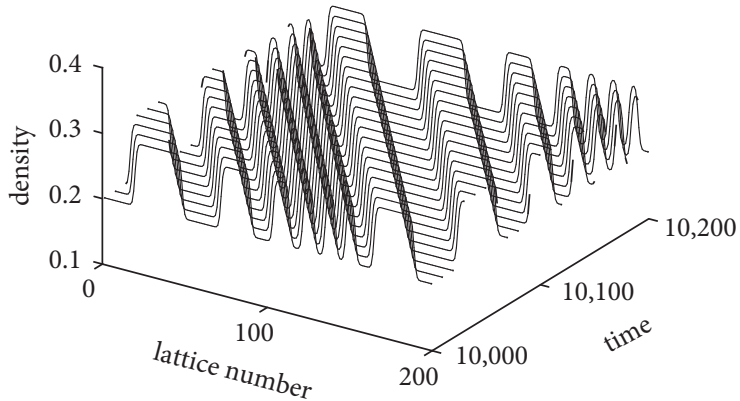

(b)

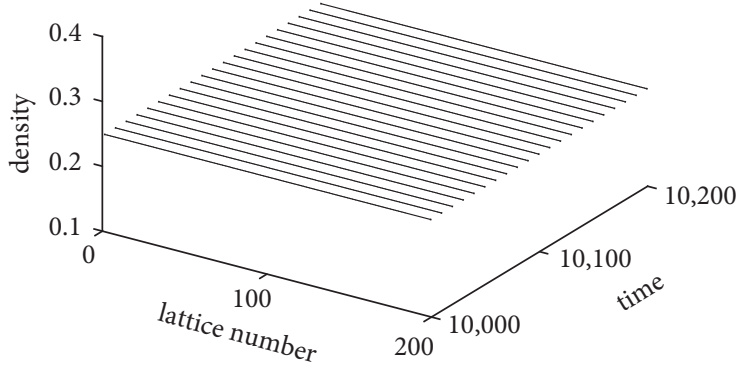

(d)

FIgURE 4: The evolution of the traffic densities for different values of parameter $\tau$ : (a) $\tau=1.0$; (b) $\tau=1.5$; (c) $\tau=2.0$; (d) $\tau=2.5$.

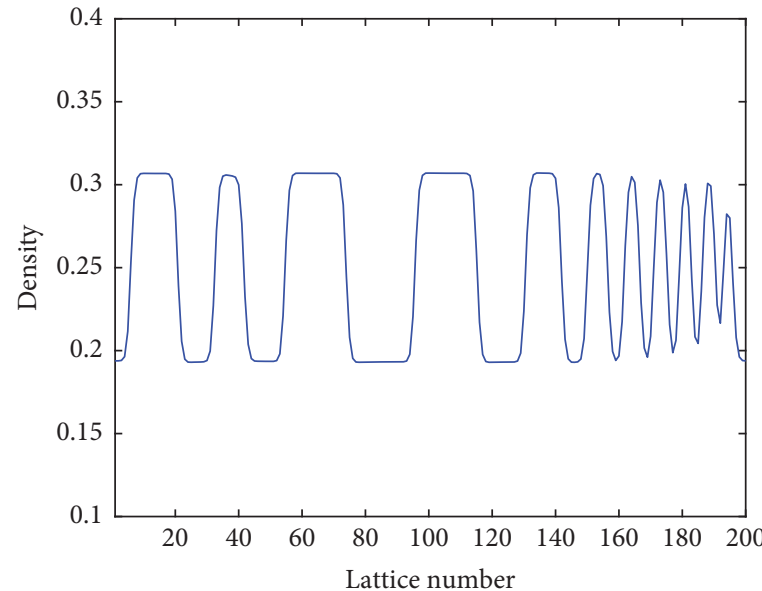

(a)

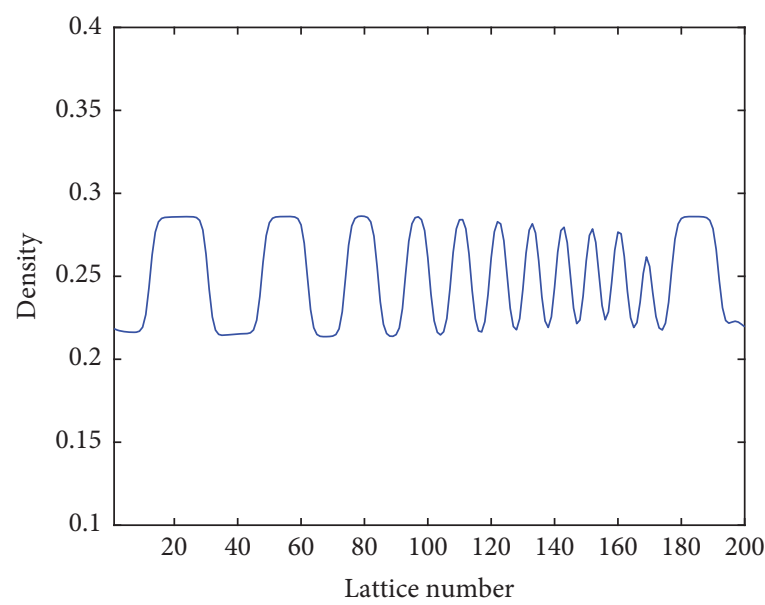

(c)

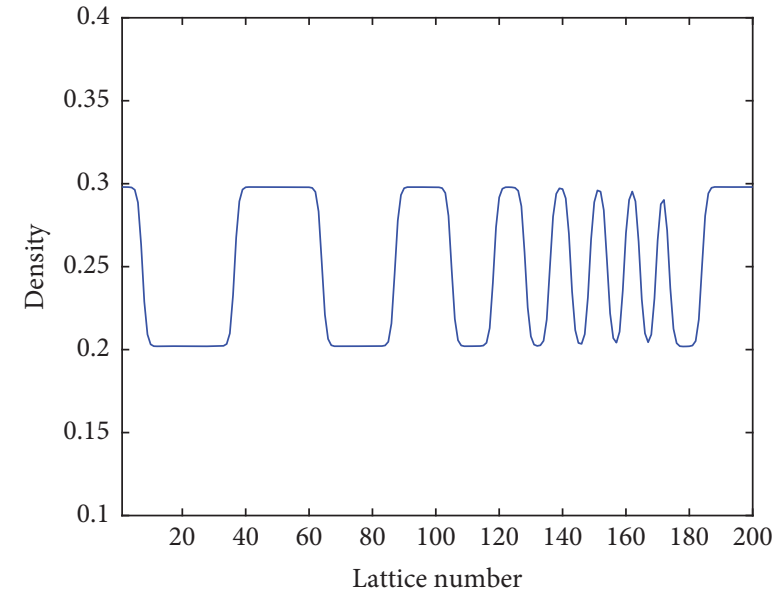

(b)

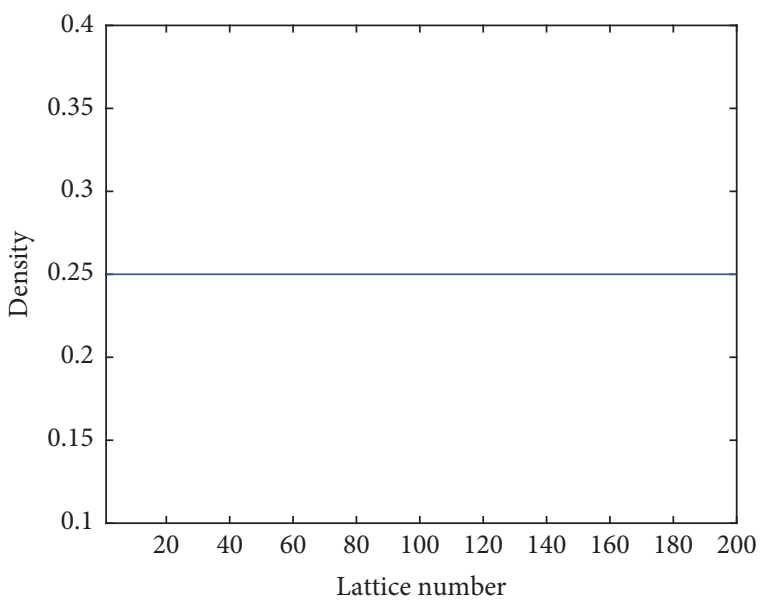

(d)

Figure 5: The density profile at $t=10200$ with different values of $\tau$ : (a) $\tau=1.0$; (b) $\tau=1.5$; (c) $\tau=2.0$; (d) $\tau=2.5$. 


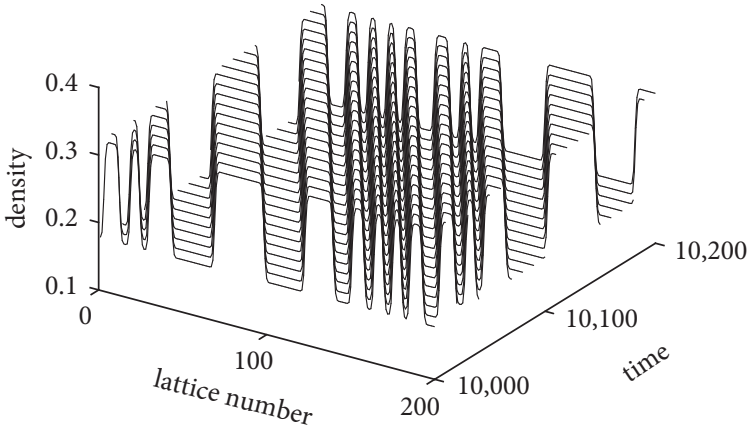

(a)

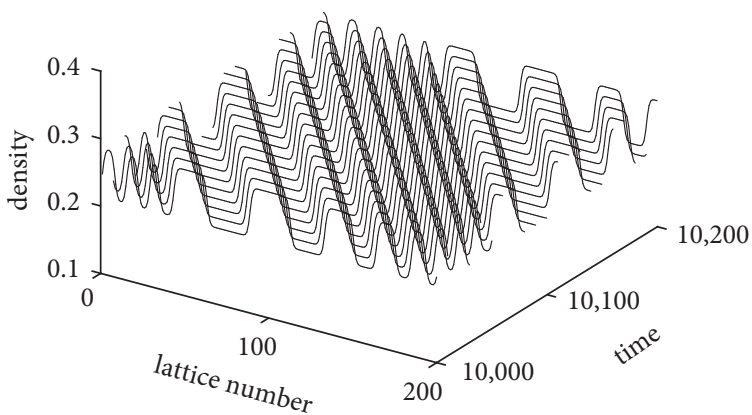

(c)

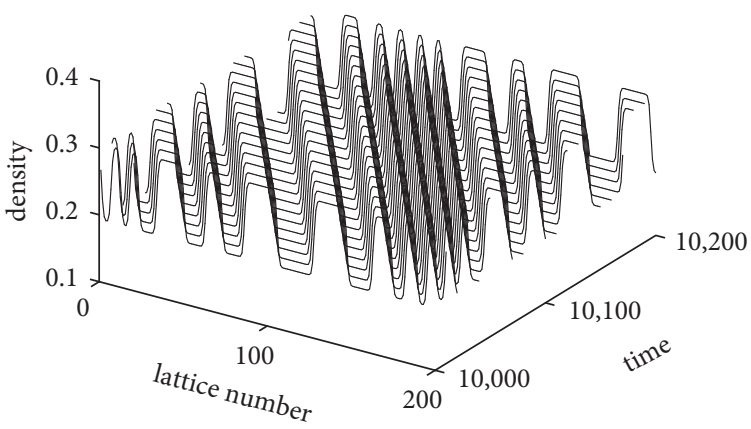

(b)

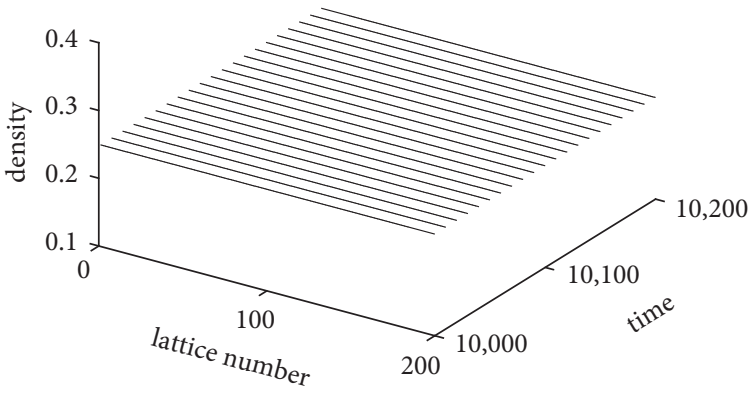

(d)

Figure 6: The evolution of the traffic densities for different values of parameter $p$ : (a) $p=0$; (b) $p=0.05$; (c) $p=0.1$; (d) $p=0.15$.

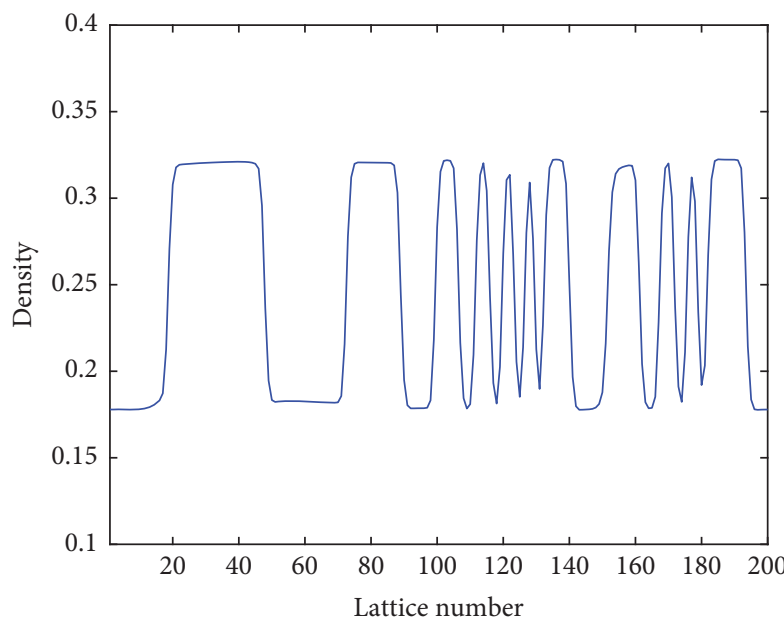

(a)

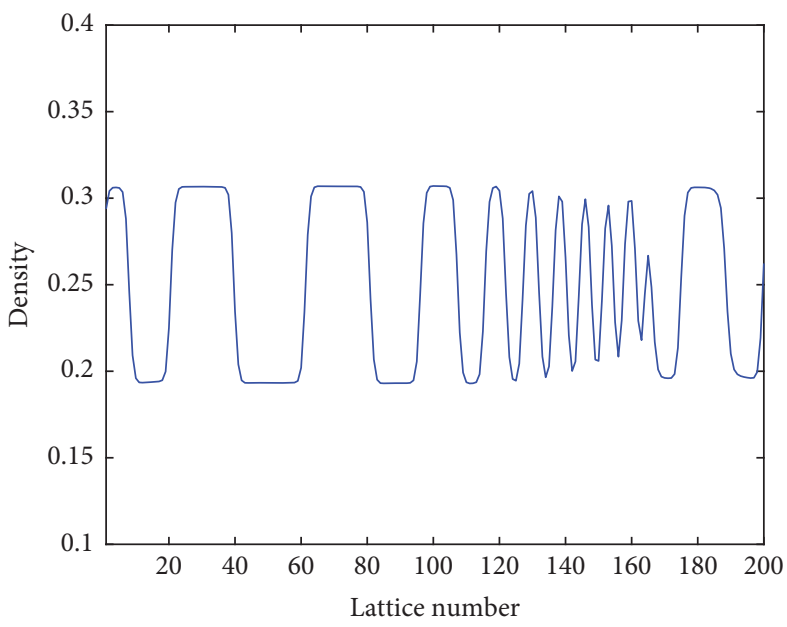

(b)

Figure 7: Continued. 


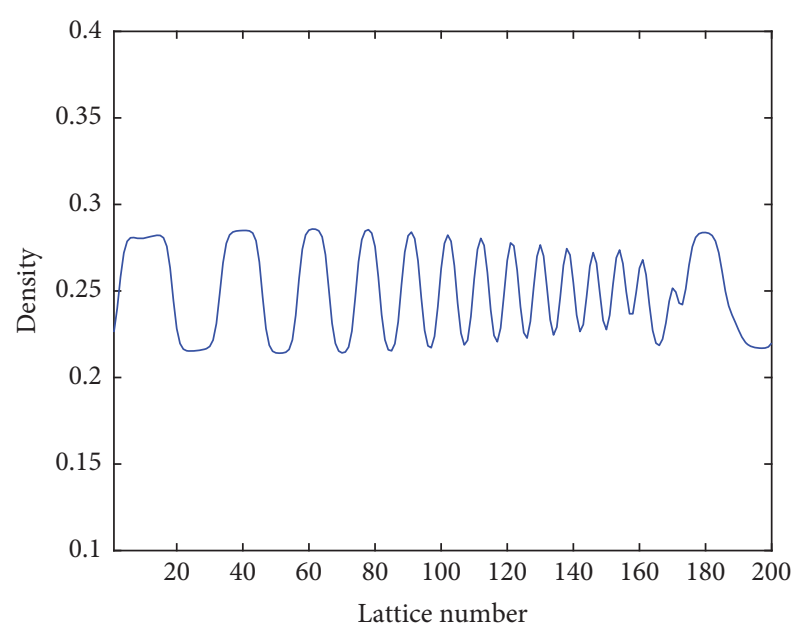

(c)

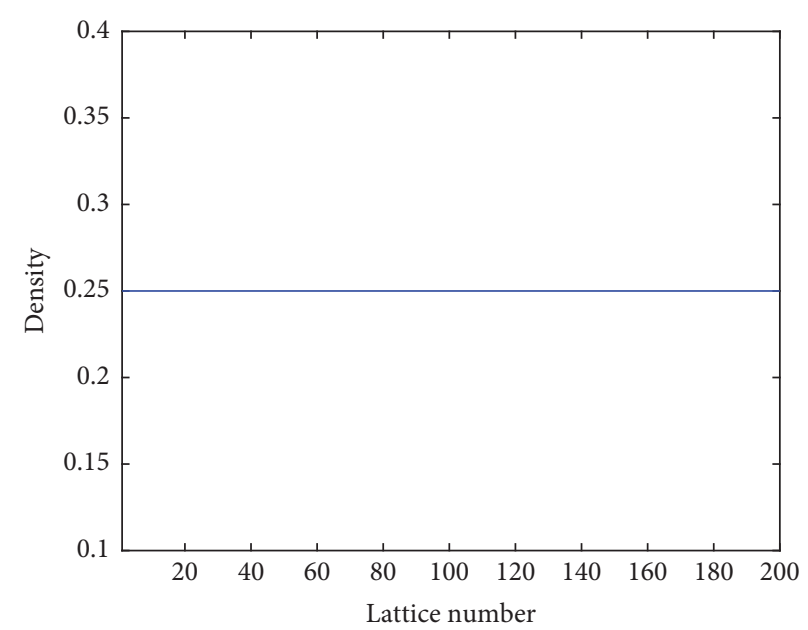

(d)

Figure 7: The density profile at $t=10200$ with different values of $p$ : (a) $p=0$; (b) $p=0.05$; (c) $p=0.1$; (d) $p=0.15$.
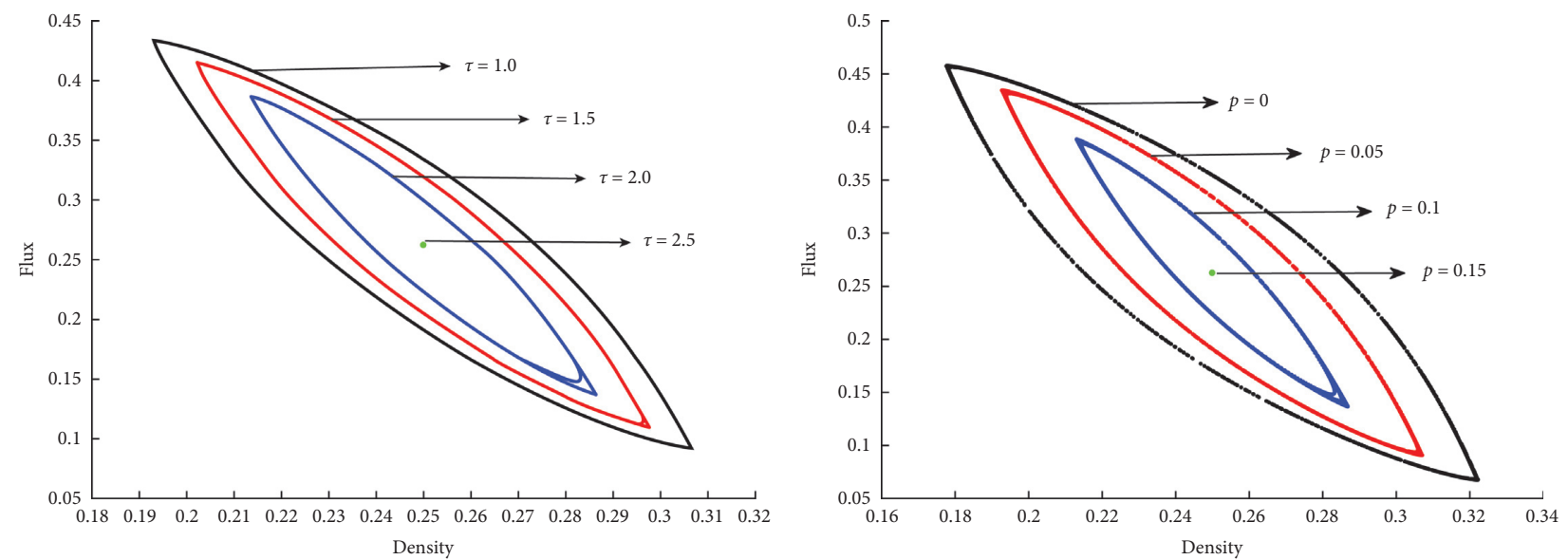

Figure 8: Hysteresis loops of traffic flux and density for (a) $\tau=1,1.5,2$, and 2.5 with fixed $\gamma=0.05$ and (b) $p=0,0.05,0.1$, and 0.15 with fixed $\gamma=0.05$.

subfigure shown in Figure 4 at $t=10200$. Moreover, Figures 5(a)-5(c) reveal that the amplitudes of density waves corresponding to Figures $4(\mathrm{a})-4(\mathrm{c})$ weaken as the anticipation time $\tau$ increases. When $\tau=2.5$, i.e., the parameters in Figure 4(d) satisfy the linear stability condition, the added perturbation will disappear, and the traffic flow will eventually return to a uniform state. Figure $5(\mathrm{~d})$ can get results similar to Figure $4(\mathrm{~d})$. This phenomenon indicates that driver's anticipation effect of flux difference could significantly promote the stabilization of traffic flow.

4.2. The Effect of Sensitivity Coefficient p. To expose the sensitivity coefficient's influence on the stability of the traffic system, the temporal and spatial evolution of density waves between time $t=10000-10200 \mathrm{~s}$ is exhibited in Figure 6 for different values of $p$ with fixed $\gamma=0.05$ and $\tau=2$. When $p=0$, the FADBE model degrades into Ge and Cheng's model [39]. Note that the parameters in Figures 6(a)-6(c) do not satisfy the linear stable condition equation (14). Therefore, the stop-go traffic density waves occur with the small added perturbation in Figures 6(a)-6(c). Meanwhile, the amplitude of the density wave decreases gradually as the value of $p$ increases. Especially in Figure 6(d), the congested state entirely disappears when $p=0.15$. The relationship between Figures 6 and 7 is similar to the relationship between Figures 4 and 5. Figure 7 describes the density profile of each subfigure shown in Figure 6 at $t=10200$. As parameter $p$ increases, the traffic flow becomes more gentle and stable. This indicates that the sensitivity coefficient $p$ contributes to suppressing the traffic congestion.

Figures 8(a) and 8(b) describe the flux against the density with different parameters corresponding to the traffic flow in Figures 4 and 6, respectively. In Figure 8, the amplitude of the hysteresis loops reduced with the increasing value of $\tau$ or $p$. When the corresponding parameter $\tau \geq 2.5$ or $p \geq 0.15$, the hysteresis loop turns into a stable point, which means that the traffic flow eventually becomes stable even with the 
added small perturbation. To sum up, as the anticipation time $\tau$ or the sensitivity coefficient $p$ increases, the stability of the system can be enhanced by incorporating the driver's anticipation effect and the "backward-looking" effect.

\section{Conclusion}

In this paper, a novel lattice hydrodynamic model with the anticipation effect of flux difference and the "backwardlooking" effect is constructed to explore the traffic driving behavior for a single-lane traffic system. The stability conditions and the solution of the $\mathrm{mKdV}$ equation are deduced, respectively, based on the linear stability analysis and nonlinear theory. The results of the theoretical analysis display that the increasing values of both the driver's anticipation time and the sensitivity coefficient of the anticipation flux difference can contribute to enlarging the stable region. Numerical simulations reveal that the FADBE model is effective in stabilizing traffic flow even with perturbations. By considering the anticipation effect of flux difference and the "backward-looking" effect simultaneously, the traffic congestion can be alleviated effectively, which is in accordance with the conclusions of the theoretical analysis. Future work will focus on solving the traffic congestion on multilane road problem, the automation for longitudinal driving, and the vehicular emergency warning system.

\section{Data Availability}

The data used to support the findings of this study are available from the corresponding author upon request.

\section{Conflicts of Interest}

The authors declare that there are no conflicts of interest regarding the publication of this paper.

\section{Acknowledgments}

This work was supported in part by the National Natural Science Foundation of China (Grant no. 61903203) and the student's innovation and entrepreneurship training program from Jiangsu Provincial Department of Education (Grant no. 202110304055Y).

\section{References}

[1] H. Zhao, H. Yue, T. Gu, and W. Li, "Cps-based reliability enhancement mechanism for vehicular emergency warning system," International Journal of Intelligent Transportation Systems Research, vol. 17, no. 3, pp. 232-241, 2019.

[2] H. Zhao, H. Yue, T. Gu, C. Li, and D. Zhou, "Low delay and seamless connectivity-based message propagation mechanism for VANET of VCPS," Wireless Personal Communications, vol. 118, no. 4, pp. 3385-3402, 2021.

[3] T. Tang, H. Huang, S. C. Wong, and R. Jiang, "A car-following model with the anticipation effect of potential lane changing," Acta Mechanica Sinica, vol. 24, no. 4, pp. 399-407, 2008.

[4] T. Tang, W. Shi, H. Shang, and Y. Wang, "A new car-following model with consideration of inter-vehicle communication," Nonlinear Dynamics, vol. 76, no. 4, pp. 2017-2023, 2014.
[5] W.-X. Zhu and L.-D. Zhang, "A new car-following model for autonomous vehicles flow with mean expected velocity field," Physica A: Statistical Mechanics and Its Applications, vol. 492, pp. 2154-2165, 2018.

[6] H. Kuang, Z.-P. Xu, X.-L. Li, and S.-M. Lo, “An extended carfollowing model accounting for the average headway effect in intelligent transportation system," Physica A: Statistical Mechanics and Its Applications, vol. 471, pp. 778-787, 2017.

[7] H. Kuang, Z. P. Xu, X. L. Li, and S. M. Lo, "An extended carfollowing model accounting for the honk effect and numerical tests," Nonlinear Dynamics, vol. 87, 2017.

[8] W.-X. Zhu and L.-D. Zhang, "Analysis of car-following model with cascade compensation strategy," Physica A: Statistical Mechanics and Its Applications, vol. 449, pp. 265-274, 2016.

[9] M. Ma, G. Ma, and S. Liang, "Density waves in car-following model for autonomous vehicles with backward looking effect," Applied Mathematical Modelling, vol. 94, 2021.

[10] H. Zhao, Q. Chen, W. Shi, T. Gu, and W. Li, "Stability analysis of an improved car-following model accounting for the driver's characteristics and automation," Physica A: Statistical Mechanics and Its Applications, vol. 526, Article ID 120990, 2019.

[11] T.-Q. Tang, Y.-X. Rui, J. Zhang, and H.-Y. Shang, “A cellular automation model accounting for bicycle's group behavior," Physica A: Statistical Mechanics and Its Applications, vol. 492, pp. 1782-1797, 2018.

[12] M. Zamith, R. C. P. Leal-Toledo, E. Clua, E. M. Toledo, and G. V. P. d. Magalhães, "A new stochastic cellular automata model for traffic flow simulation with drivers' behavior prediction," Journal of Computational Science, vol. 9, pp. 51-56, 2015.

[13] R. Cheng, H. Ge, and J. Wang, "An extended macro traffic flow model accounting for multiple optimal velocity functions with different probabilities," Physics Letters A, vol. 381, no. 32, pp. 2608-2620, 2017.

[14] R. Cheng, H. Ge, and J. Wang, "KdV-Burgers equation in a new continuum model based on full velocity difference model considering anticipation effect," Physica A: Statistical Mechanics and Its Applications, vol. 481, 2017.

[15] H. Liu, P. Zheng, K. Zhu, and H. Ge, "KdV-Burgers equation in the modified continuum model considering anticipation effect," Physica A: Statistical Mechanics and Its Applications, vol. 438, pp. 26-31, 2015.

[16] G. Zhang, D.-H. Sun, H. Liu, and D. Chen, "Stability analysis of a new lattice hydrodynamic model by considering lattice's self-anticipative density effect," Physica A: Statistical Mechanics and Its Applications, vol. 486, pp. 806-813, 2017.

[17] G. Zhang, "The self-stabilization effect of lattice's historical flow in a new lattice hydrodynamic model," Nonlinear Dynamics, vol. 91, no. 2, pp. 809-817, 2018.

[18] G. Peng, "A new lattice model of traffic flow with the consideration of individual difference of anticipation driving behavior," Communications in Nonlinear Science and Numerical Simulation, vol. 18, no. 10, pp. 2801-2806, 2013.

[19] Y. Zhang, M. Zhao, D. Sun, Z. Zhou, and C. Dong, "A new feedback control scheme for the lattice hydrodynamic model with drivers' sensory memory," International Journal of Modern Physics C, vol. 32, 2021.

[20] Z. Li, X. Li, and F. Liu, "Stabilization analysis and modified $\mathrm{KdV}$ equation of lattice models with consideration of relative current," International Journal of Modern Physics C, vol. 19, no. 8, pp. 1163-1173, 2008.

[21] H. Zhao, D. Xia, S. Yang, and G. Peng, "The delayed-time effect of traffic flux on traffic stability for two-lane freeway," 
Physica A: Statistical Mechanics and Its Applications, vol. 540, Article ID 123066, 2020.

[22] C. Zhai and W. Wu, "Designing continuous delay feedback control for lattice hydrodynamic model under cyber-attacks and connected vehicle environment," Communications in Nonlinear Science and Numerical Simulation, vol. 95, Article ID 105667, 2021.

[23] G. Zhang and G.-H. Peng, "Research on the stabilization effect of continuous self-delayed traffic flux in macro traffic modeling," Physica A: Statistical Mechanics and Its Applications, vol. 526, Article ID 121012, 2019.

[24] G. Zhang, Y. Zhang, D.-B. Pan, and R.-J. Huang, "Study on the continuous delayed optimal flow on traffic stability in a new macro traffic model," Physica A: Statistical Mechanics and Its Applications, vol. 534, Article ID 122029, 2019.

[25] D. Helbing and M. Treiber, "Gas-kinetic-based traffic model explaining observed hysteretic phase transition," Physical Review Letters, vol. 81, pp. 3042-3045, 1999.

[26] T. Nagatani, "Modified KdV equation for jamming transition in the continuum models of traffic," Physica A: Statistical Mechanics and Its Applications, vol. 261, no. 3-4, pp. 599-607, 1998.

[27] G. Peng, F. Nie, B. Cao, and C. Liu, “A driver's memory lattice model of traffic flow and its numerical simulation," Nonlinear Dynamics, vol. 67, no. 3, pp. 1811-1815, 2012.

[28] Q. Zhai, H. Ge, and R. Cheng, "An extended continuum model considering optimal velocity change with memory and numerical tests," Physica A: Statistical Mechanics and its Applications, vol. 490, pp. 774-785, 2018.

[29] S. Qin, Z. He, and R. Cheng, "An extended lattice hydrodynamic model based on control theory considering the memory effect of flux difference," Physica A: Statistical Mechanics and Its Applications, vol. 509, pp. 809-816, 2018.

[30] C. Liu, Y. He, and G. Peng, "The impact of the density difference memory integral on traffic stability in two-lane lattice hydrodynamic model," Physica A: Statistical Mechanics and Its Applications, vol. 532, Article ID 121750, 2019.

[31] Q. Wang, R. Cheng, and H. Ge, "A novel lattice hydrodynamic model accounting for driver's memory effect and the difference of optimal velocity on curved road," Physica A: Statistical Mechanics and Its Applications, vol. 559, Article ID 125023, 2020.

[32] X. Li, K. Fang, and G. Peng, “A new lattice model accounting for multiple optimal current differences' anticipation effect in two-lane system," Physica A: Statistical Mechanics and Its Applications, vol. 486, pp. 814-826, 2017.

[33] A. K. Gupta and P. Redhu, "Analyses of driver's anticipation effect in sensing relative flux in a new lattice model for twolane traffic system," Physica A: Statistical Mechanics and Its Applications, vol. 392, no. 22, pp. 5622-5632, 2013.

[34] D.-H. Sun, G. Zhang, M. Zhao, S.-L. Cheng, and J.-D. Cao, "Stability analysis of feedforward anticipation optimal flux difference in traffic lattice hydrodynamic theory," Communications in Nonlinear Science and Numerical Simulation, vol. 56, pp. 287-295, 2018.

[35] T. Wang, Z. Gao, J. Zhang, and X. Zhao, "A new lattice hydrodynamic model for two-lane traffic with the consideration of density difference effect," Nonlinear Dynamics, vol. $75,2014$.

[36] C. Jiang, R. Cheng, and H. Ge, "An improved lattice hydrodynamic model considering the "backward looking" effect and the traffic interruption probability," Nonlinear Dynamics, vol. 91, no. 2, pp. 777-784, 2018.
[37] G. Peng, W. Lu, and H. He, "Impact of the traffic interruption probability of optimal current on traffic congestion in lattice model," Physica A: Statistical Mechanics and Its Applications, vol. 425, pp. 27-33, 2015.

[38] D.-H. Sun, G. Zhang, W.-N. Liu, M. Zhao, S.-L. Cheng, and T. Zhou, "Effect of explicit lane changing in traffic lattice hydrodynamic model with interruption," Nonlinear Dynamics, vol. 86, pp. 269-282, 2016.

[39] H.-X. Ge and R.-J. Cheng, "The "backward looking" effect in the lattice hydrodynamic model," Physica A: Statistical Mechanics and Its Applications, vol. 387, no. 28, pp. 6952-6958, 2008.

[40] Q. Wang and H. Ge, "An improved lattice hydrodynamic model accounting for the effect of "backward looking" and flow integral," Physica A: Statistical Mechanics and Its Applications, vol. 513, pp. 438-446, 2019.

[41] X. Qi, H. Ge, and R. Cheng, "Analysis of a novel lattice hydrodynamic model considering density integral and "backward looking" effect," Physica A: Statistical Mechanics and Its Applications, vol. 525, pp. 714-723, 2019.

[42] A. K. Gupta and P. Redhu, "Analyses of the driver's anticipation effect in a new lattice hydrodynamic traffic flow model with passing," Nonlinear Dynamics, vol. 76, no. 2, pp. 1001-1011, 2014.

[43] T. Wang, R. Zang, K. Xu, and J. Zhang, "Analysis of predictive effect on lattice hydrodynamic traffic flow model," Physica A: Statistical Mechanics and Its Applications, vol. 526, Article ID 120711, 2019.

[44] D. Kaur and S. Sharma, "A new two-lane lattice model by considering predictive effect in traffic flow," Physica A: Statistical Mechanics and Its Applications, vol. 539, Article ID 122913, 2020.

[45] S. Sharma, "Effect of driver's anticipation in a new two-lane lattice model with the consideration of optimal current difference," Nonlinear Dynamics, vol. 81, no. 1-2, pp. 991-1003, 2015.

[46] P. Redhu and A. K. Gupta, "Effect of forward looking sites on a multi-phase lattice hydrodynamic model," Physica A: Statistical Mechanics and Its Applications, vol. 445, pp. 150-160, 2016.

[47] Y. Chang and R. Cheng, "Effect of speed deviation and anticipation effect of flux difference in the lattice hydrodynamic model," Physica A: Statistical Mechanics and Its Applications, vol. 531, Article ID 121751, 2019. 